\title{
Existence of Periodic Solutions in a Discrete Predator-Prey System with Beddington-DeAngelis Functional Responses
}

\author{
Changjin $\mathrm{Xu}^{1}$ and Maoxin Liao ${ }^{2}$ \\ ${ }^{1}$ Guizhou Key Laboratory of Economics System Simulation, School of Mathematics and Statistics, \\ Guizhou College of Finance and Economics, Guiyang 550004, China \\ ${ }^{2}$ School of Mathematics and Physics, Nanhua University, Hengyang 421001, China
}

Correspondence should be addressed to Changjin Xu, xcj403@126.com

Received 8 July 2011; Accepted 27 October 2011

Academic Editor: Aloys Krieg

Copyright (C) 2011 C. Xu and M. Liao. This is an open access article distributed under the Creative Commons Attribution License, which permits unrestricted use, distribution, and reproduction in any medium, provided the original work is properly cited.

A discrete predator-prey model with Holling II and Beddington-DeAngelis functional responses is investigated. With the aid of differential equations with piecewise constant arguments, a discrete version of continuous nonautonomous delayed predator-prey model with BeddingtonDeAngelis functional responses is proposed. By using Gaines and Mawhin's continuation theorem of coincidence degree theory, sufficient conditions for the existence of positive solutions of the model are established.

\section{Introduction}

In population dynamics, the functional response refers to the number of prey eaten per predator per unit time as a function of prey density. Based on a lot of experiments on mammals, Holling [1] proposed three kinds of functional responses as follows:
(1) $p_{1}(x)=a x$,
(2) $p_{2}(x)=a x /(a+x)$,
(3) $p_{3}(x)=a x^{2} /\left(a+x^{2}\right)$,

where $x$ represents the density of prey. Functions $p_{1}(x), p_{2}(x)$, and $p_{3}(x)$ refer to Holling types I, II, and III, respectively, and $a>0$ is the predation rate of the predator. After that, the dynamical properties of predator-prey systems with functional response have received great attention from both theoretical and mathematical biologists; for example, Liu et al. [2] 
investigated the coexistence of predators and preys of the following predator-prey system with Holling II functional response:

$$
\begin{gathered}
\frac{d x}{d t}=r x(t)\left[1-\frac{x(t)}{K}\right]-\frac{a x(t) y(t)}{1+b x(t)}-\frac{A x(t) z(t)}{1+B x(t)}, \\
\frac{d y}{d t}=y(t)\left[-d+\frac{e x(t)}{1+b x(t)}\right], \\
\frac{d z}{d t}=z(t)\left[-D+\frac{E x(t)}{1+B x(t)}\right],
\end{gathered}
$$

where $x(t)$ stands for the density of the prey, $y(t)$ and $z(t)$ are the densities of the predators, respectively, and $r, a, A, d, e, D, E, K, b$, and $B$ are positive constants. Assuming that one predator consumes prey according to the Holling II functional response and the other predator consumes prey according to the Beddington-DeAngelis functional response, Cantrell et al. [3] proposed the revised version of system (1.1) as follows:

$$
\begin{gathered}
\frac{d x}{d t}=r x(t)\left[1-\frac{x(t)}{K}\right]-\frac{a x(t) y(t)}{1+b x(t)}-\frac{A x(t) z(t)}{1+B x(t)+C z(t)} \\
\frac{d y}{d t}=y(t)\left[-d+\frac{e x(t)}{1+b x(t)}\right] \\
\frac{d z}{d t}=z(t)\left[-D+\frac{E x(t)}{1+B x(t)+C z(t)}\right]
\end{gathered}
$$

By using dynamical system technique and the geometrical singular perturbation theory, Cantrell et al. [3] made a discussion on the coexistence of predators and prey of system (1.2) which occurred along a stable positive equilibrium. Ko and Ryu [4] discussed the existence, stability, and uniqueness of coexistence states and the extinction and permanence of a diffusive two-competing-prey and one-predator system with Beddington-DeAngelis functional response. Chen et al. [5] analyzed the extinction of the predator and the global asymptotic stability of the boundary solution of a nonautonomous predator-prey system with the Beddington-DeAngelis functional response. Huo et al. [6] studied the global existence of positive periodic solutions for a delayed predator-prey model with the BeddingtonDeAngelis functional response. For more knowledge about this topic, one can see $[7,8]$.

Recently, Song and Li [9] investigated the following two-prey one-predator model, where two prey are competitive and the predator has Holling functional II functional response:

$$
\begin{aligned}
& \frac{d x_{1}}{d t}=x_{1}(t)\left[b_{1}-x_{1}(t)-\alpha x_{2}(t)-\frac{\eta x_{3}(t)}{1+\omega_{1} x_{1}(t)}\right], \\
& \frac{d x_{2}}{d t}=x_{2}(t)\left[b_{2}-x_{2}(t)-\beta x_{1}(t)-\frac{\mu x_{3}(t)}{1+\omega_{2} x_{2}(t)}\right], \\
& \frac{d x_{3}}{d t}=x_{3}(t)\left[-b_{3}+\frac{d \eta x_{1}(t)}{1+\omega_{1} x_{1}(t)}+\frac{d \mu x_{2}(t)}{1+\omega_{2} x_{2}(t)}\right],
\end{aligned}
$$


where $x_{1}(t)$ and $x_{2}(t)$ stand for the population size of prey (pest) species and $x_{3}(t)$ is the population size of the predator (natural species) species; $b_{i}>0(i=1,2,3)$ are intrinsic rates of increase or decrease, $\alpha>0$ and $\beta>0$ are parameters representing competitive effects between two prey, $\eta>0, \mu>0$ are positive constants, $\eta x_{1}(t) /\left(1+\omega_{1} x_{1}(t)\right)$ and $\mu x_{2}(t) /(1+$ $\left.\omega_{2} x_{2}(t)\right)$ are the Holling II functional responses. $d>0$ is the rate of conversing prey into predator.

To model mutual interference among predators, Beddington [10] andDeAngelis et al. [11] argued that the well-known Holling type II functional response will be replaced by the Beddington-DeAngelis functional responses which is similar to the Holling type II functional response but has an extra term $v_{i} x_{3}(i=1,2)$ in the denominator, then system (1.3) may be modified as the following predator-prey system with Beddington-DeAngelis functional responses:

$$
\begin{gathered}
\frac{d x_{1}}{d t}=x_{1}(t)\left[b_{1}-x_{1}(t)-\alpha x_{2}(t)-\frac{\eta x_{3}(t)}{1+\omega_{1} x_{1}(t)+v_{1} x_{3}(t)}\right], \\
\frac{d x_{2}}{d t}=x_{2}(t)\left[b_{2}-x_{2}(t)-\beta x_{1}(t)-\frac{\mu x_{3}(t)}{1+\omega_{2} x_{2}(t)+v_{2} x_{3}(t)}\right], \\
\frac{d x_{3}}{d t}=x_{3}(t)\left[-b_{3}+\frac{d \eta x_{1}(t)}{1+\omega_{1} x_{1}(t)+v_{1} x_{3}(t)}+\frac{d \mu x_{2}(t)}{1+\omega_{2} x_{2}(t)+v_{2} x_{3}(t)}\right],
\end{gathered}
$$

where $x_{1}(t)$ and $x_{2}(t)$ stand for the population size of prey (pest) species and $x_{3}(t)$ is the population size of the predator (natural species) species; $b_{i}>0(i=1,2,3)$ are intrinsic rates of increase or decrease, $\alpha>0$ and $\beta>0$ are parameters representing competitive effects between two prey, $\eta>0, \mu>0$ are positive constants, $\eta x_{3}(t) /\left(1+\omega_{1} x_{1}(t)+\nu_{1} x_{3}(t)\right)$, $\mu x_{3}(t) /\left(1+\omega_{2} x_{2}(t)+v_{2} x_{3}(t)\right), \eta x_{1}(t) /\left(1+\omega_{1} x_{1}(t)+v_{1} x_{3}(t)\right)$, and $\mu x_{2}(t) /\left(1+\omega_{2} x_{2}(t)+v_{2} x_{3}(t)\right)$ are the Beddington-DeAngelis functional responses. $d>0$ is the rate of conversing prey into predator.

In the natural word, any biological and environmental parameters are naturally subject to fluctuation in time. The effect of a periodically varying environment is important for evolutionary theory as the selective forces on systems in a fluctuating environment differ from those in a stable environment. Thus, assumptions of periodicity of parameters are a way of incorporating periodicity of the environment, such as seasonal effect of weather, food supplies, and mating habits [12]. Based on the point of view, the modification of (1.4) according to the environmental variation is the nonautonomous differential equations

$$
\begin{gathered}
\frac{d x_{1}}{d t}=x_{1}(t)\left[b_{1}(t)-x_{1}(t)-\alpha(t) x_{2}(t)-\frac{\eta(t) x_{3}(t)}{1+\omega_{1}(t) x_{1}(t)+v_{1}(t) x_{3}(t)}\right], \\
\frac{d x_{2}}{d t}=x_{2}(t)\left[b_{2}(t)-x_{2}(t)-\beta(t) x_{1}(t)-\frac{\mu(t) x_{3}(t)}{1+\omega_{2}(t) x_{2}(t)+v_{2}(t) x_{3}(t)}\right], \\
\frac{d x_{3}}{d t}=x_{3}(t)\left[-b_{3}(t)+\frac{d(t) \eta(t) x_{1}(t)}{1+\omega_{1}(t) x_{1}(t)+v_{1}(t) x_{3}(t)}+\frac{d(t) \mu(t) x_{2}(t)}{1+\omega_{2}(t) x_{2}(t)+v_{2}(t) x_{3}(t)}\right] .
\end{gathered}
$$

As is known to us, discrete time models governed by difference equations are more appropriate to describe the dynamics relationship among populations than continuous ones when the populations have nonoverlapping generations. Moreover, discrete time models can 
also provide efficient models of continuous ones for numerical simulations. Therefore, it is reasonable and interesting to study discrete time systems governed by difference equations. Recently, there are some papers which deal with these topics see [13-22]. The principle object of this article is to propose a discrete analogue system (1.5) and explore its dynamics.

The remainder of the paper is organized as follows: in Section 2, with the help of differential equations with piecewise constant arguments, we first propose a discrete analogue of system (1.5), modelling the dynamics of time nonautonomous predator-prey system with Beddington-DeAngelis functional responses, where populations have nonoverlapping generations. In Section 3, based on the coincidence degree and the related continuation theorem, sufficient conditions for the existence of positive solutions of the model are obtained.

\section{A Discrete Version of Model (1.5)}

There are several different ways of deriving discrete time version of dynamical systems corresponding to continuous time formulations. One of the ways of deriving difference equations modelling the dynamics of populations with nonoverlapping generations that we will use in the following is based on appropriate modifications of models with overlapping generations. For more details about the approach, we refer to $[15,18]$.

In the following, we will discrete the system (1.5). Assume that the average growth rates in system (1.5) change at regular intervals of time, then we can obtain the following modified system:

$$
\begin{aligned}
\frac{1}{x_{1}(t)} \dot{x}_{1}(t)= & b_{1}([t])-x_{1}([t])-\alpha([t]) x_{2}([t])-\frac{\eta([t]) x_{3}([t])}{1+\omega_{1}([t]) x_{1}([t])+v_{1}([t]) x_{3}([t])} \\
\frac{1}{x_{2}(t)} \dot{x}_{2}(t)= & b_{2}([t])-x_{2}([t])-\beta([t]) x_{1}([t])-\frac{\mu([t]) x_{3}([t])}{1+\omega_{2}([t]) x_{2}([t])+v_{2}([t]) x_{3}([t])} \\
\frac{1}{x_{3}(t)} \dot{x}_{3}(t)= & -b_{3}([t])+\frac{d([t]) \eta([t]) x_{1}([t])}{1+\omega_{1}([t]) x_{1}([t])+v_{1}([t]) x_{3}([t])} \\
& +\frac{d([t]) \mu([t]) x_{2}([t])}{1+\omega_{2}([t]) x_{2}([t])+v_{2}([t]) x_{3}([t])}
\end{aligned}
$$

where $[t]$ denotes the integer part of $t, t \in(0,+\infty)$ and $t \neq 0,1,2, \ldots$ Equations of type (2.1) are known as differential equations with piecewise constant arguments, and these equations occupy a position midway between differential equations and difference equations. By a solution of (2.1), we mean a function $x=\left(x_{1}, x_{2}, x_{3}\right)^{T}$, which is defined for $t \in[0,+\infty)$ and has the following properties:

(1) $x$ is continuous on $[0,+\infty)$;

(2) the derivative $d x / d t=\left(d x_{1}(t) / d t, d x_{2}(t) / d t, d x_{3}(t) / d t\right)^{T}$ exists at each point $t \in$ $[0,+\infty)$ with the possible exception of the points $t \in\{0,1,2, \ldots\}$, where left-sided derivative exists;

(3) The equations in (2.1) are satisfied on each interval $[k, k+1)$ with $k=0,1,2, \ldots$ 
We integrate (2.1) on any interval of the form $[k, k+1), k=0,1,2, \ldots$, and obtain for $k \leq t<k+1, k=0,1,2, \ldots$,

$$
\begin{aligned}
& x(t)=x(k) \exp \left\{\left[b_{1}(k)-x_{1}(k)-\alpha(k) x_{2}(k)-\frac{\eta(k) x_{3}(k)}{1+\omega_{1}(k) x_{1}(k)+v_{1}(k) x_{3}(k)}\right](t-k)\right\}, \\
& y(t)=y(k) \exp \left\{\left[b_{2}(k)-x_{2}(k)-\beta(k) x_{1}(k)-\frac{\mu(k) x_{3}(k)}{1+\omega_{2}(k) x_{2}(k)+v_{2}(k) x_{3}(k)}\right](t-k)\right\}, \\
& z(t)=z(k) \exp \left\{\left[-b_{3}(k)+\frac{d(k) \eta(k) x_{1}(k)}{1+\omega_{1}(k) x_{1}(k)+v_{1}(k) x_{3}(k)}+\frac{d(k) \mu(k) x_{2}(k)}{1+\omega_{2}(k) x_{2}(k)+v_{2}(k) x_{3}(k)}\right](t-k)\right\} .
\end{aligned}
$$

Let $t \rightarrow k+1$, then (2.2) takes the following form:

$$
\begin{aligned}
& x(k+1)=x(k) \exp \left\{b_{1}(k)-x_{1}(k)-\alpha(k) x_{2}(k)-\frac{\eta(k) x_{3}(k)}{1+\omega_{1}(k) x_{1}(k)+v_{1}(k) x_{3}(k)}\right\}, \\
& y(k+1)=y(k) \exp \left\{b_{2}(k)-x_{2}(k)-\beta(k) x_{1}(k)-\frac{\mu(k) x_{3}(k)}{1+\omega_{2}(k) x_{2}(k)+v_{2}(k) x_{3}(k)}\right\}, \\
& z(k+1)=z(k) \exp \left\{-b_{3}(k)+\frac{d(k) \eta(k) x_{1}(k)}{1+\omega_{1}(k) x_{1}(k)+v_{1}(k) x_{3}(k)}+\frac{d(k) \mu(k) x_{2}(k)}{1+\omega_{2}(k) x_{2}(k)+v_{2}(k) x_{3}(k)}\right\},
\end{aligned}
$$

which is a discrete time analogue of system (1.5), where $k=0,1,2, \ldots$

\section{Existence of Positive Periodic Solutions}

For convenience and simplicity in the following discussion, we always use the notations below throughout the paper:

$$
I_{\omega}:=\{0,1,2, \ldots, \omega-1\}, \quad \bar{f}:=\frac{1}{\omega} \sum_{k=0}^{\omega-1} f(k), \quad f^{L}=\min _{k \in I_{\omega}}\{f(k)\}, \quad f^{M}=\max _{k \in I_{\omega}}\{f(k)\},
$$

where $f(k)$ is an $\omega$-periodic sequence of real numbers defined for $k \in Z$. For system (2.3), we always assume that

(H1) $b_{i}(i=1,2,3), d, \alpha, \beta, \eta, \mu, \omega_{j}, v_{j}(j=1,2): Z \rightarrow R^{+}$are $\omega$-periodic.

In order to explore the existence of positive periodic solutions of (2.3) and for the reader's convenience, we will first summarize below a few concepts and results without proof, borrowing from [23].

Let $X, Y$ be normed vector spaces, $L:$ Dom $L \subset X \rightarrow Y$ is a linear mapping, $N: X \rightarrow$ $Y$ is a continuous mapping. The mapping $L$ will be called a Fredholm mapping of index zero if $\operatorname{dim} \operatorname{Ker} L=\operatorname{codim} \operatorname{Im} L<+\infty$ and $\operatorname{Im} L$ is closed in $Y$. If $L$ is a Fredholm mapping of index zero and there exist continuous projectors $P: X \rightarrow X$ and $Q: Y \rightarrow Y$ such that 
$\operatorname{Im} P=\operatorname{Ker} L, \operatorname{Im} L=\operatorname{Ker} Q=\operatorname{Im}(I-Q)$, it follows that $L \mid \operatorname{Dom} L \cap \operatorname{Ker} P:(I-P) X \rightarrow \operatorname{Im} L$ is invertible. We denote the inverse of that map by $K_{P}$. If $\Omega$ is an open bounded subset of $X$, the mapping $N$ will be called $L$ compact on $\bar{\Omega}$ if $Q N(\bar{\Omega})$ is bounded and $K_{P}(I-Q) N: \bar{\Omega} \rightarrow X$ is compact. Since $\operatorname{Im} Q$ is isomorphic to $\operatorname{Ker} L$, there exist isomorphisms $J: \operatorname{Im} Q \rightarrow \operatorname{Ker} L$.

Lemma 3.1 (see Continuation Theorem [23]). Let L be a Fredholm mapping of index zero, and let $N$ be L compact on $\bar{\Omega}$. Suppose that,

(a) for each $\lambda \in(0,1)$, every solution $x$ of $L x=\lambda N x$ is such that $x \notin \partial \Omega$;

(b) $Q N x \neq 0$ for each $x \in \operatorname{Ker} L \cap \partial \Omega$, and $\operatorname{deg}\{J Q N, \Omega \cap \operatorname{Ker} L, 0\} \neq 0$,

then the equation $L x=N x$ has at least one solution lying in $\operatorname{Dom} L \cap \bar{\Omega}$.

Lemma 3.2 (see [15]). Let $g: Z \rightarrow R$ be $\omega$-periodic, that is, $g(k+\omega)=g(k)$. Then for any fixed $k_{1}, k_{2} \in I_{\omega}$ and any $k \in Z$, one has

$$
\begin{aligned}
& g(k) \leq g\left(k_{1}\right)+\sum_{s=0}^{\omega-1}|g(s+1)-g(s)|, \\
& g(k) \geq g\left(k_{2}\right)-\sum_{s=0}^{\omega-1}|g(s+1)-g(s)| .
\end{aligned}
$$

Define

$$
l_{3}=\left\{u=\{u(k)\}: u(k) \in R^{3}, k \in Z\right\} .
$$

For $a=\left(a_{1}, a_{2}, a_{3}\right)^{T} \in R^{3}$, define $|a|=\max \left\{\left|a_{1}\right|,\left|a_{2}\right|,\left|a_{3}\right|\right\}$. Let $l^{\omega} \subset l_{3}$ denote the subspace of all $\omega$-periodic sequences equipped with the usual supremum norm $\|\cdot\|$, that is, $\|u\|=\max _{k \in I_{\omega}}|u(k)|$, for any $u=\{u(k): k \in Z\} \in l^{\omega}$. It is easy to show that $l_{\omega}$ is a finite-dimensional Banach space.

Let

$$
\begin{gathered}
l_{0}^{\omega}=\left\{u=\{u(k)\} \in l^{\omega}: \sum_{k=0}^{\omega-1} u(k)=0\right\}, \\
l_{c}^{\omega}=\left\{u=\{u(k)\} \in l^{\omega}: u(k)=h \in R^{3}, k \in Z\right\} .
\end{gathered}
$$

Then it follows that $l_{0}^{\omega}$ and $l_{c}^{\omega}$ are both closed linear subspaces of $l^{\omega}$ and

$$
l^{\omega}=l_{0}^{\omega}+l_{c}^{\omega}, \quad \operatorname{dim} l_{c}^{\omega}=3
$$


In the following, we will be ready to establish our result.

Theorem 3.3. Let $S_{1}, S_{1}^{\prime}, S_{2}, S_{2}^{\prime}, k_{0}$, and $h_{0}$ be defined by (3.30), (3.50), (3.34), (3.46), (3.39), and (3.55), respectively. In addition to the condition (H1), assume further that the following conditions:

(H2) $\bar{b}_{3}>\max \left\{\overline{d \eta} \exp \left\{S_{1}\right\}, \overline{d \mu} \exp \left\{S_{2}^{\prime}\right\}\right\}, \eta^{L}>b_{1}^{L} v_{1}^{M}$,

(H3) $k_{0}<0, h_{0}<0$

hold. Then system (2.3) has at least an w-periodic solution.

Proof. Let $u_{1}(k)=\ln \left[x_{1}(k)\right], u_{2}(k)=\ln \left[x_{2}(k)\right]$, and $u_{3}(k)=\ln \left[x_{3}(k)\right]$. Then (2.3) takes the form

$$
\begin{aligned}
& u_{1}(k+1)-u_{1}(k)=f_{1}(k), \\
& u_{2}(k+1)-u_{2}(k)=f_{2}(k), \\
& u_{3}(k+1)-u_{3}(k)=f_{3}(k),
\end{aligned}
$$

where

$$
\begin{aligned}
f_{1}(k)= & b_{1}(k)-\exp \left\{u_{1}(k)\right\}-\alpha(k) \exp \left\{u_{2}(k)\right\}-\frac{\eta(k) \exp \left\{u_{3}(k)\right\}}{1+\omega_{1}(k) \exp \left\{u_{1}(k)\right\}+v_{1}(k) \exp \left\{u_{3}(k)\right\}}, \\
f_{2}(k)= & b_{2}(k)-\exp \left\{u_{2}(k)\right\}-\beta(k) \exp \left\{u_{1}(k)\right\}-\frac{\mu(k) \exp \left\{u_{3}(k)\right\}}{1+\omega_{2}(k) \exp \left\{u_{2}(k)\right\}+v_{2}(k) \exp \left\{u_{3}(k)\right\}}, \\
f_{3}(k)= & -b_{3}(k)+\frac{d(k) \eta(k) \exp \left\{u_{1}(k)\right\}}{1+\omega_{1}(k) \exp \left\{u_{1}(k)\right\}+v_{1}(k) \exp \left\{u_{3}(k)\right\}} \\
& +\frac{d(k) \mu(k) \exp \left\{u_{2}(k)\right\}}{1+\omega_{2}(k) \exp \left\{u_{2}(k)\right\}+v_{2}(k) \exp \left\{u_{3}(k)\right\}} .
\end{aligned}
$$

Let $X=Y=l^{\omega}$,

$$
\begin{gathered}
(L u)(k)=u(k+1)-u(k)=\left(\begin{array}{l}
u_{1}(k+1)-u_{1}(k) \\
u_{2}(k+1)-u_{2}(k) \\
u_{3}(k+1)-u_{3}(k)
\end{array}\right) \\
(N u)(k)=\left(\begin{array}{c}
f_{1}(k) \\
f_{2}(k) \\
f_{3}(k)
\end{array}\right)
\end{gathered}
$$

where $u \in X, k \in Z$. Then it is trivial to see that $L$ is a bounded linear operator and

$$
\operatorname{Ker} L=l_{c}^{\omega}, \quad \operatorname{Im} L=l_{0}^{\omega},
$$

$\operatorname{dim} \operatorname{Ker} L=3=\operatorname{codim} \operatorname{Im} L$. 
Then it follows that $L$ is a Fredholm mapping of index zero. Define

$$
P u=\frac{1}{\omega} \sum_{s=0}^{\omega-1} u(s), \quad u \in X, \quad Q v=\frac{1}{\omega} \sum_{s=0}^{\omega-1} v(s), \quad v \in Y .
$$

It is not difficult to show that $P$ and $Q$ are continuous projectors such that

$$
\operatorname{Im} P=\operatorname{Ker} L, \quad \operatorname{Im} L=\operatorname{Ker} Q=\operatorname{Im}(I-Q) .
$$

Furthermore, the generalized inverse (to $L) K_{P}: \operatorname{Im} L \rightarrow \operatorname{Ker} P \bigcap \operatorname{Dom} L$ exists and is given by

$$
K_{P}(v)=\sum_{s=0}^{\omega-1} v(s)-\frac{1}{\omega} \sum_{s=0}^{\omega-1}(\omega-s) v(s)
$$

Obviously, $Q N$ and $K_{P}(I-Q) N$ are continuous. Since $X$ is a finite-dimensional Banach space, using the Ascoli-Arzela theorem, it is not difficult to show that $\overline{K_{P}(I-Q) N(\bar{\Omega})}$ is compact for any open bounded set $\Omega \subset X$. Moreover, $Q N(\bar{\Omega})$ is bounded. Thus, $N$ is $L$-compact on $\bar{\Omega}$ with any open bounded set $\Omega \subset X$.

Now we are at the point to search for an appropriate open, bounded subset $\Omega$ for the application of the continuation theorem. Corresponding to the operator equation $L u=\lambda N u$, $\lambda \in(0,1)$, we have

$$
\begin{aligned}
& u_{1}(k+1)-u_{1}(k)=\lambda f_{1}(k), \\
& u_{2}(k+1)-u_{2}(k)=\lambda f_{2}(k), \\
& u_{3}(k+1)-u_{3}(k)=\lambda f_{3}(k) .
\end{aligned}
$$

Suppose that $u(k)=\left(u_{1}(k), u_{2}(k), u_{3}(k)\right)^{T} \in X$ is an arbitrary solution of system (3.14) for a certain $\lambda \in(0,1)$. Summing both sides of (3.14) from 0 to $\omega-1$ with respect to $k$, respectively, we obtain

$$
\begin{aligned}
& \sum_{k=0}^{\omega-1}\left[\exp \left\{u_{1}(k)\right\}+\alpha(k) \exp \left\{u_{2}(k)\right\}+\frac{\eta(k) \exp \left\{u_{3}(k)\right\}}{1+\omega_{1}(k) \exp \left\{u_{1}(k)\right\}+v_{1}(k) \exp \left\{u_{3}(k)\right\}}\right]=\bar{b}_{1} \omega, \\
& \sum_{k=0}^{\omega-1}\left[\exp \left\{u_{2}(k)\right\}+\beta(k) \exp \left\{u_{1}(k)\right\}+\frac{\mu(k) \exp \left\{u_{3}(k)\right\}}{1+\omega_{2}(k) \exp \left\{u_{2}(k)\right\}+v_{2}(k) \exp \left\{u_{3}(k)\right\}}\right]=\bar{b}_{2} \omega, \\
& \sum_{k=0}^{\omega-1}\left[\frac{d(k) \eta(k) \exp \left\{u_{1}(k)\right\}}{1+\omega_{1}(k) \exp \left\{u_{1}(k)\right\}+v_{1}(k) \exp \left\{u_{3}(k)\right\}}+\frac{d(k) \mu(k) \exp \left\{u_{2}(k)\right\}}{1+\omega_{2}(k) \exp \left\{u_{2}(k)\right\}+v_{2}(k) \exp \left\{u_{3}(k)\right\}}\right]=\bar{b}_{3} \omega .
\end{aligned}
$$


It follows from (3.14) and(3.15) that

$$
\begin{aligned}
& \sum_{k=0}^{\omega-1}\left|u_{1}(k+1)-u_{1}(k)\right| \leq 2 \bar{b}_{1} \omega, \\
& \sum_{k=0}^{\omega-1}\left|u_{2}(k+1)-u_{2}(k)\right| \leq 2 \bar{b}_{2} \omega, \\
& \sum_{k=0}^{\omega-1}\left|u_{3}(k+1)-u_{3}(k)\right| \leq 2 \bar{b}_{3} \omega .
\end{aligned}
$$

In view of the hypothesis that $u=\{u(k)\} \in X$, there exist $\xi_{i}, \eta_{i} \in I_{\omega}$ such that

$$
u_{i}\left(\xi_{i}\right)=\min _{k \in I_{\omega}}\left\{u_{i}(k)\right\}, \quad u_{i}\left(\eta_{i}\right)=\max _{k \in I_{\omega}}\left\{u_{i}(k)\right\} \quad(i=1,2,3) .
$$

Then it is obvious that

$$
\nabla u_{i}\left(\eta_{i}\right) \leq 0, \quad \nabla u_{i}\left(\xi_{i}\right) \geq 0, \quad i=1,2,3,
$$

where $\nabla$ denotes the forward difference operator $\nabla u(k)=u(k+1)-u(k)$.

In view of (3.8), we get

$$
\begin{aligned}
& b_{1}\left(\xi_{1}\right)-\exp \left\{u_{1}\left(\xi_{1}\right)\right\}-\alpha(k) \exp \left\{u_{2}\left(\xi_{1}\right)\right\}-\frac{\eta\left(\xi_{1}\right) \exp \left\{u_{3}\left(\xi_{1}\right)\right\}}{1+\omega_{1}\left(\xi_{1}\right) \exp \left\{u_{1}\left(\xi_{1}\right)\right\}+\nu_{1}\left(\xi_{1}\right) \exp \left\{u_{3}\left(\xi_{1}\right)\right\}} \geq 0 \\
& b_{2}\left(\xi_{2}\right)-\exp \left\{u_{2}\left(\xi_{2}\right)\right\}-\beta\left(\xi_{2}\right) \exp \left\{u_{1}\left(\xi_{2}\right)\right\}-\frac{\mu\left(\xi_{2}\right) \exp \left\{u_{3}\left(\xi_{2}\right)\right\}}{1+\omega_{2}\left(\xi_{2}\right) \exp \left\{u_{2}\left(\xi_{2}\right)\right\}+v_{2}\left(\xi_{2}\right) \exp \left\{u_{3}\left(\xi_{2}\right)\right\}} \geq 0
\end{aligned}
$$

Then we have

$$
\exp \left\{u_{1}\left(\xi_{1}\right)\right\}<b_{1}\left(\xi_{1}\right), \quad \exp \left\{u_{2}\left(\xi_{2}\right)\right\}<b_{2}\left(\xi_{2}\right),
$$

which leads to

$$
u_{1}\left(\xi_{1}\right)<\ln b_{1}\left(\xi_{1}\right)=\ln \left[b_{1}^{L}\right], \quad u_{2}\left(\xi_{2}\right)<\ln b_{2}\left(\xi_{2}\right)=\ln \left[b_{2}^{L}\right]
$$


From (3.16), we have

$$
\sum_{k=0}^{\omega-1}\left[d(k) \eta(k) \exp \left\{u_{1}\left(\eta_{1}\right)\right\}+d(k) \mu(k) \exp \left\{u_{2}\left(\eta_{2}\right)\right\}\right]>\bar{b}_{3} \omega
$$

that is,

$$
\overline{d \eta} \exp \left\{u_{1}\left(\eta_{1}\right)\right\}+\overline{d \mu} \exp \left\{u_{2}\left(\eta_{2}\right)\right\}>\bar{b}_{3}
$$

In the sequel, we consider two cases.

Case 1. If $u_{1}\left(\eta_{1}\right) \geq u_{2}\left(\eta_{2}\right)$, then it follows from (3.26) that

$$
\overline{d \eta} \exp \left\{u_{1}\left(\eta_{1}\right)\right\}+\overline{d \mu} \exp \left\{u_{1}\left(\eta_{1}\right)\right\}>\bar{b}_{3}
$$

which leads to

$$
u_{1}\left(\eta_{1}\right)>\ln \left[\frac{\bar{b}_{3}}{\overline{d(\eta+\mu)}}\right]
$$

In view of Lemma 3.2, (3.16), (3.24), and (3.28), we have

$$
\begin{aligned}
u_{1}(k) & \leq u_{1}\left(\xi_{1}\right)+\sum_{s=0}^{\omega-1}\left|u_{1}(s+1)-u_{1}(s)\right| \\
& \leq \ln \left[b_{1}^{L}\right]+2 \bar{b}_{1} \omega:=b_{1}, \\
u_{1}(k) & \geq u_{1}\left(\eta_{1}\right)-\sum_{s=0}^{\omega-1}\left|u_{1}(s+1)-u_{1}(s)\right| \\
& \geq \ln \left[\frac{\bar{b}_{3}}{\overline{d(\eta+\mu)}}\right]-2 \bar{b}_{1} \omega:=B_{1} .
\end{aligned}
$$

It follows from (3.29) that

$$
\max _{k \in I_{\omega}}\left\{u_{1}(k)\right\}<\max \left\{\left|b_{1}\right|,\left|B_{1}\right|\right\}:=S_{1} .
$$

By (3.26), it is easy to obtain that

$$
\overline{d \eta} \exp \left\{S_{1}\right\}+\overline{d \mu} \exp \left\{u_{2}\left(\eta_{2}\right)\right\}>\bar{b}_{3}
$$


International Journal of Mathematics and Mathematical Sciences

Hence,

$$
u_{2}\left(\eta_{2}\right)>\ln \left[\frac{\bar{b}_{3}-\overline{d \eta} \exp \left\{S_{1}\right\}}{\overline{d \mu}}\right]
$$

In view of Lemma 3.2, (3.18), (3.24), and (3.32), we have

$$
\begin{aligned}
u_{2}(k) & \leq u_{2}\left(\xi_{2}\right)+\sum_{s=0}^{\omega-1}\left|u_{2}(s+1)-u_{2}(s)\right| \\
& \leq \ln \left[b_{2}^{L}\right]+2 \bar{b}_{2} \omega:=b_{2} \\
u_{2}(k) & \geq u_{2}\left(\eta_{1}\right)-\sum_{s=0}^{\omega-1}\left|u_{2}(s+1)-u_{2}(s)\right| \\
& \geq \ln \left[\frac{\bar{b}_{3}-\overline{d \eta} \exp \left\{S_{1}\right\}}{\overline{d \mu}}\right]-2 \bar{b}_{2} \omega:=B_{2} .
\end{aligned}
$$

It follows from (3.33) that

$$
\max _{k \in I_{\omega}}\left\{u_{2}(k)\right\}<\max \left\{\left|b_{2}\right|,\left|B_{2}\right|\right\}:=S_{2} .
$$

Noticing that $\eta\left(\xi_{1}\right) \exp \left\{u_{3}\right\} /\left(1+\omega_{1}\left(\xi_{1}\right) \exp \left\{u_{1}\left(\xi_{1}\right)\right\}+v_{1}\left(\xi_{1}\right) \exp \left\{u_{3}\right\}\right)$ is an increasing function with respect to $u_{3}$, from (3.21), we have

$$
\begin{aligned}
b_{1}^{L}=b_{1}\left(\xi_{1}\right) & >\frac{\eta\left(\xi_{1}\right) \exp \left\{u_{3}\left(\xi_{1}\right)\right\}}{1+\omega_{1}\left(\xi_{1}\right) \exp \left\{u_{1}\left(\xi_{1}\right)\right\}+v_{1}\left(\xi_{1}\right) \exp \left\{u_{3}\left(\xi_{1}\right)\right\}} \\
& \geq \frac{\eta^{L} \exp \left\{u_{3}\left(\xi_{3}\right)\right\}}{1+\omega_{1}^{M} \exp \left\{S_{1}\right\}+v_{1}^{M} \exp \left\{u_{3}\left(\xi_{3}\right)\right\}} .
\end{aligned}
$$

Hence,

$$
u_{3}\left(\xi_{3}\right)<\ln \left[\frac{b_{1}^{L}\left(1+\omega_{1}^{M} \exp \left\{S_{1}\right\}\right)}{\eta^{L}-b_{1}^{L} v_{1}^{M}}\right]
$$

From (3.16), we get

$$
\sum_{k=0}^{\omega-1}\left[\frac{d(k) \eta(k) \exp \left\{-S_{1}\right\}}{1+\omega_{1}^{M} \exp \left\{S_{1}\right\}+v_{1}^{M} \exp \left\{u_{3}\left(\eta_{3}\right)\right\}}+\frac{d(k) \mu(k) \exp \left\{-S_{2}\right\}}{1+\omega_{2}^{M} \exp \left\{S_{2}\right\}+v_{2}^{M} \exp \left\{u_{3}\left(\eta_{3}\right)\right\}}\right] \leq \bar{b}_{3} \omega
$$


Then,

$$
k_{2} \exp \left\{2 u_{3}\left(\eta_{3}\right)\right\}+k_{1} \exp \left\{u_{3}\left(\eta_{3}\right)\right\}+k_{0} \geq 0,
$$

where

$$
\begin{aligned}
k_{0}= & \bar{b}_{3}\left(1+\omega_{1}^{M} \exp \left\{S_{1}\right\}\right)\left(1+\omega_{2}^{M} \exp \left\{S_{2}\right\}\right) \\
& -\overline{d \eta} \exp \left\{-S_{1}\right\}\left(1+\omega_{2}^{M} \exp \left\{S_{2}\right\}\right) \\
& -\overline{d \mu} \exp \left\{-S_{2}\right\}\left(1+\omega_{1}^{M} \exp \left\{S_{1}\right\}\right), \\
k_{1}= & \bar{b}_{3}\left[v_{2}^{M}\left(1+\omega_{1}^{M} \exp \left\{S_{1}\right\}\right)+v_{1}^{M}\left(1+\omega_{2}^{M} \exp \left\{S_{2}\right\}\right)\right] \\
& -\left[\overline{d \eta} v_{2}^{M} \exp \left\{-S_{1}\right\}+\overline{d \mu} v_{1}^{M} \exp \left\{-S_{2}\right\}\right], \\
k_{2}= & \bar{b}_{3} v_{1}^{M} v_{2}^{M} .
\end{aligned}
$$

Thus,

$$
u_{3}\left(\eta_{3}\right) \geq \ln \left[\frac{-k_{1}+\sqrt{k_{1}^{2}-k_{2} k_{0}}}{2 k_{2}}\right]
$$

In view of Lemma 3.2, (3.18), (3.36), and (3.40), we have

$$
\begin{aligned}
u_{3}(k) & \leq u_{3}\left(\xi_{3}\right)+\sum_{s=0}^{\omega-1}\left|u_{3}(s+1)-u_{3}(s)\right| \\
& \leq \ln \left[\frac{b_{1}^{L}\left(1+\omega_{1}^{M} \exp \left\{S_{1}\right\}\right)}{\eta^{L}-b_{1}^{L} v_{1}^{M}}\right]+2 \bar{r} \omega:=b_{3}, \\
u_{3}(k) & \geq u_{3}\left(\eta_{3}\right)-\sum_{s=0}^{\omega-1}\left|u_{3}(s+1)-u_{3}(s)\right| \\
& \geq \ln \left[\frac{-k_{1}+\sqrt{k_{1}^{2}-k_{2} k_{0}}}{2 k_{2}}\right]-2 \bar{b}_{3} \omega:=B_{3} .
\end{aligned}
$$

It follows from (3.41) that

$$
\max _{k \in I_{\omega}}\left\{u_{3}(k)\right\}<\max \left\{\left|b_{3}\right|,\left|B_{3}\right|\right\}:=S_{3} .
$$


International Journal of Mathematics and Mathematical Sciences

Case 2. If $u_{1}\left(\eta_{1}\right)<u_{2}\left(\eta_{2}\right)$, then it follows from (3.26) that

$$
\overline{d \eta} \exp \left\{u_{2}\left(\eta_{2}\right)\right\}+\overline{d \mu} \exp \left\{u_{2}\left(\eta_{2}\right)\right\}>\bar{b}_{3}
$$

which leads to

$$
u_{2}\left(\eta_{2}\right)>\ln \left[\frac{\bar{b}_{3}}{\overline{d(\eta+\mu)}}\right]
$$

In view of Lemma 3.2, (3.17), (3.24), and (3.44), we have

$$
\begin{aligned}
u_{2}(k) & \leq u_{2}\left(\xi_{2}\right)+\sum_{s=0}^{\omega-1}\left|u_{2}(s+1)-u_{2}(s)\right| \\
& \leq \ln \left[b_{2}^{L}\right]+2 \bar{b}_{2} \omega:=b_{2}^{\prime}, \\
u_{2}(k) & \geq u_{2}\left(\eta_{2}\right)-\sum_{s=0}^{\omega-1}\left|u_{2}(s+1)-u_{2}(s)\right| \\
& \geq \ln \left[\frac{\bar{b}_{3}}{\overline{d(\eta+\mu)}}\right]-2 \bar{b}_{2} \omega:=B_{2}^{\prime} .
\end{aligned}
$$

Combining both equation of (3.45), one obtains

$$
\max _{k \in I_{\omega}}\left\{u_{2}(k)\right\}<\max \left\{\left|b_{2}^{\prime}\right|,\left|B_{2}^{\prime}\right|\right\}:=S_{2}^{\prime}
$$

By (3.26), it is easy to obtain that

$$
\overline{d \eta} \exp \left\{u_{1}\left(\eta_{1}\right)\right\}+\overline{d \mu} \exp \left\{S_{2}^{\prime}\right\}>\bar{b}_{3}
$$

Hence,

$$
u_{1}\left(\eta_{1}\right)>\ln \left[\frac{\bar{b}_{3}-\overline{d \mu} \exp \left\{S_{2}^{\prime}\right\}}{\overline{d \eta}}\right]
$$


By virtue of Lemma 3.2, (3.16), (3.24), and (3.48), we have

$$
\begin{aligned}
u_{1}(k) & \leq u_{1}\left(\xi_{1}\right)+\sum_{s=0}^{\omega-1}\left|u_{1}(s+1)-u_{1}(s)\right| \\
& \leq \ln \left[b_{1}^{L}\right]+2 \bar{b}_{2} \omega:=b_{1}^{\prime} \\
u_{1}(k) \geq & u_{1}\left(\eta_{1}\right)-\sum_{s=0}^{\omega-1}\left|u_{1}(s+1)-u_{1}(s)\right| \\
& \geq \ln \left[\frac{\bar{b}_{3}-\overline{d \mu} \exp \left\{S_{2}^{\prime}\right\}}{\overline{d \eta}}\right]-2 \bar{b}_{1} \omega:=B_{1}^{\prime} .
\end{aligned}
$$

It follows from (3.49) that

$$
\max _{k \in I_{\omega}}\left\{u_{1}(k)\right\}<\max \left\{\left|b_{1}^{\prime}\right|,\left|B_{1}^{\prime}\right|\right\}:=S_{1}^{\prime}
$$

Considering that $\eta\left(\xi_{1}\right) \exp \left\{u_{3}\right\} /\left(1+\omega_{1}\left(\xi_{1}\right) \exp \left\{u_{1}\left(\xi_{1}\right)\right\}+v_{1}\left(\xi_{1}\right) \exp \left\{u_{3}\right\}\right)$ is an increasing function with respect to $u_{3}$, from (3.21), we have

$$
\begin{aligned}
b_{1}^{L}=b_{1}\left(\xi_{1}\right) & >\frac{\eta\left(\xi_{1}\right) \exp \left\{u_{3}\left(\xi_{1}\right)\right\}}{1+\omega_{1}\left(\xi_{1}\right) \exp \left\{u_{1}\left(\xi_{1}\right)\right\}+v_{1}\left(\xi_{1}\right) \exp \left\{u_{3}\left(\xi_{1}\right)\right\}} \\
& \geq \frac{\eta^{L} \exp \left\{u_{3}\left(\xi_{3}\right)\right\}}{1+\omega_{1}^{M} \exp \left\{S_{1}^{\prime}\right\}+v_{1}^{M} \exp \left\{u_{3}\left(\xi_{3}\right)\right\}} .
\end{aligned}
$$

Therefore,

$$
u_{3}\left(\xi_{3}\right)<\ln \left[\frac{b_{1}^{L}\left(1+\omega_{1}^{M} \exp \left\{S_{1}^{\prime}\right\}\right)}{\eta^{L}-b_{1}^{L} v_{1}^{M}}\right]
$$

From (3.16), we know that

$$
\sum_{s=0}^{\omega-1}\left[\frac{d(k) \eta(k) \exp \left\{-S_{1}^{\prime}\right\}}{1+\omega_{1}^{M} \exp \left\{S_{1}^{\prime}\right\}+v_{1}^{M} \exp \left\{u_{3}\left(\eta_{3}\right)\right\}}+\frac{d(k) \mu(k) \exp \left\{-S_{2}^{\prime}\right\}}{1+\omega_{2}^{M} \exp \left\{S_{2}^{\prime}\right\}+v_{2}^{M} \exp \left\{u_{3}\left(\eta_{3}\right)\right\}}\right] \leq \bar{b}_{3} \omega
$$

Then,

$$
h_{2} \exp \left\{2 u_{3}\left(\eta_{3}\right)\right\}+h_{1} \exp \left\{u_{3}\left(\eta_{3}\right)\right\}+h_{0} \geq 0,
$$


International Journal of Mathematics and Mathematical Sciences

where

$$
\begin{aligned}
h_{0}= & \bar{b}_{3}\left(1+\omega_{1}^{M} \exp \left\{S_{1}^{\prime}\right\}\right)\left(1+\omega_{2}^{M} \exp \left\{S_{2}^{\prime}\right\}\right) \\
& -\overline{d \eta} \exp \left\{-S_{1}^{\prime}\right\}\left(1+\omega_{2}^{M} \exp \left\{S_{2}^{\prime}\right\}\right) \\
& -\overline{d \mu} \exp \left\{-S_{2}^{\prime}\right\}\left(1+\omega_{1}^{M} \exp \left\{S_{1}^{\prime}\right\}\right), \\
h_{1}= & \bar{b}_{3}\left[v_{2}^{M}\left(1+\omega_{1}^{M} \exp \left\{S_{1}^{\prime}\right\}\right)+v_{1}^{M}\left(1+\omega_{2}^{M} \exp \left\{S_{2}^{\prime}\right\}\right)\right] \\
& -\left[\overline{d \eta} v_{2}^{M} \exp \left\{-S_{1}^{\prime}\right\}+\overline{d \mu} v_{1}^{M} \exp \left\{-S_{2}^{\prime}\right\}\right], \\
h_{2}= & \bar{b}_{3} v_{1}^{M} v_{2}^{M} .
\end{aligned}
$$

Thus,

$$
u_{3}\left(\eta_{3}\right) \geq \ln \left[\frac{-h_{1}+\sqrt{h_{1}^{2}-h_{2} h_{0}}}{2 h_{2}}\right]
$$

In view of Lemma 3.2, (3.18), (3.52), and (3.56), we have

$$
\begin{aligned}
u_{3}(k) & \leq u_{3}\left(\xi_{3}\right)+\sum_{s=0}^{\omega-1}\left|u_{3}(s+1)-u_{3}(s)\right| \\
& \leq \ln \left[\frac{b_{1}^{L}\left(1+\omega_{1}^{M} \exp \left\{S_{1}\right\}\right)}{\eta^{L}-b_{1}^{L} v_{1}^{M}}\right]+2 \bar{r} \omega:=b_{3}^{\prime}, \\
u_{3}(k) & \geq u_{3}\left(\eta_{3}\right)-\sum_{s=0}^{\omega-1}\left|u_{3}(s+1)-u_{3}(s)\right| \\
& \geq \ln \left[\frac{-k_{1}+\sqrt{k_{1}^{2}-k_{2} k_{0}}}{2 k_{2}}\right]-2 \bar{b}_{3} \omega:=B_{3}^{\prime} .
\end{aligned}
$$

It follows from (3.57) that

$$
\max _{k \in I_{\omega}}\left\{u_{3}(k)\right\}<\max \left\{\left|b_{3}^{\prime}\right|,\left|B_{3}^{\prime}\right|\right\}:=S_{3}^{\prime}
$$


Obviously, $S_{1}, S_{2}, S_{2}^{\prime}, S_{3}$, and $S_{3}^{\prime}$ are independent of $\lambda \in(0,1)$. Take $M=S_{1}+\max \left\{S_{2}, S_{2}^{\prime}\right\}+$ $\max \left\{S_{3}, S_{3}^{\prime}\right\}+M_{0}$, where $M_{0}$ is taken sufficiently large such that each solution $\left(u_{1}^{*}, u_{2}^{*}, u_{3}^{*}\right)^{T}$ of the following algebraic equations:

$$
\begin{aligned}
& \bar{b}_{1}-\exp \left(u_{1}\right)-\bar{\alpha} \exp \left(u_{2}\right)-\frac{1}{\omega} \sum_{k=0}^{\omega-1}\left[\frac{\eta(k) \exp \left(u_{3}\right)}{1+\omega_{1}(k) \exp \left(u_{1}\right)+v_{1}(k) \exp \left(u_{3}\right)}\right]=0, \\
& \bar{b}_{2}-\exp \left(u_{2}\right)-\bar{\beta} \exp \left(u_{1}\right)-\frac{1}{\omega} \sum_{k=0}^{\omega-1}\left[\frac{\mu(k) \exp \left(u_{3}\right)}{1+\omega_{2}(k) \exp \left(u_{2}\right)+v_{2}(k) \exp \left(u_{3}\right)}\right]=0, \\
& -\bar{b}_{3}+\frac{1}{\omega} \sum_{k=0}^{\omega-1}\left[\frac{d(k) \eta(k) \exp \left(u_{1}\right)}{1+\omega_{1}(k) \exp \left(u_{1}\right)+v_{1}(k) \exp \left(u_{3}\right)}\right]+\frac{1}{\omega} \sum_{k=0}^{\omega-1}\left[\frac{d(k) \mu(k) \exp \left(u_{2}\right)}{1+\omega_{2}(k) \exp \left(u_{2}\right)+v_{2}(k) \exp \left(u_{3}\right)}\right]=0
\end{aligned}
$$

satisfies $\max \left\{\left|u_{1}^{*}\right|,\left|u_{2}^{*}\right|,\left|u_{3}^{*}\right|\right\}<M_{0}$.

Now we have proved that any solution $u=\{u(k)\}=\left\{\left(u_{1}(k), u_{2}(k), u_{3}(k)\right)^{T}\right\}$ of (3.6) in $X$ satisfies $\|u\|<M, k \in Z$.

Let $\Omega:=\{u=\{u(k)\} \in X:\|u\|<M\}$. Then it is easy to see that $\Omega$ is an open, bounded set in $X$ and verifies requirement (a) of Lemma 3.1. When $u \in \partial \Omega \cap \operatorname{Ker} L, u=\left\{\left(u_{1}, u_{2}, u_{3}\right)^{T}\right\}$ is a constant vector in $R^{3}$ with $\|u\|=\max \left\{\left|u_{1}\right|,\left|u_{2}\right|,\left|u_{3}\right|\right\}=M$. Then

$$
\mathrm{QNu}=\left(\begin{array}{c}
\bar{b}_{1}-\exp \left(u_{1}\right)-\bar{\alpha} \exp \left(u_{2}\right)-\frac{1}{\omega} \sum_{k=0}^{\omega-1}\left[\frac{\eta(k) \exp \left(u_{3}\right)}{1+\omega_{1}(k) \exp \left(u_{1}\right)+v_{1}(k) \exp \left(u_{3}\right)}\right] \\
\bar{b}_{2}-\exp \left(u_{2}\right)-\bar{\beta} \exp \left(u_{1}\right)-\frac{1}{\omega} \sum_{k=0}^{\omega-1}\left[\frac{\mu(k) \exp (z)}{1+\omega_{2}(k) \exp \left(u_{2}\right)+v_{2}(k) \exp \left(u_{3}\right)}\right] \\
-\bar{b}_{3}+\frac{1}{\omega} \sum_{k=0}^{\omega-1}\left[\frac{d(k) \eta(k) \exp \left(u_{1}\right)}{1+\omega_{1}(k) \exp \left(u_{1}\right)+v_{1}(k) \exp \left(u_{3}\right)}\right] \\
+\frac{1}{\omega} \sum_{k=0}^{\omega-1}\left[\frac{d(k) \mu(k) \exp \left(u_{2}\right)}{1+\omega_{2}(k) \exp \left(u_{2}\right)+v_{2}(k) \exp \left(u_{3}\right)}\right]
\end{array}\right) \neq 0 .
$$

Define the homotopy $\phi: \operatorname{Dom} L \times[0,1] \rightarrow X$ by $\phi\left(u_{1}, u_{2}, u_{3}, \mu\right)=\mu Q N u+(1-\mu) G u, \mu \in[0,1]$, where

$$
G u=\frac{1}{\omega} \sum_{k=0}^{\omega-1}\left(\begin{array}{c}
\bar{b}_{1}-\exp \left(u_{1}\right) \\
\bar{b}_{2}-\exp \left(u_{2}\right) \\
-\bar{b}_{3}+\frac{1}{\omega} \sum_{k=0}^{\omega-1}\left[\frac{d(k) \eta(k) \exp \left(u_{1}\right)}{1+\omega_{1}(k) \exp \left(u_{1}\right)+v_{1}(k) \exp \left(u_{3}\right)}\right]
\end{array}\right)
$$


Let $J$ be the identity mapping. According to the definition of topology, direct calculation yields

$$
\begin{aligned}
\operatorname{deg}\{ & \left.J Q N\left(u_{1}, u_{2}, u_{3}\right)^{T} ; \Omega \cap \operatorname{ker} L ; 0\right\} \\
= & \operatorname{deg}\left\{Q N\left(u_{1}, u_{2}, u_{3}\right)^{T} ; \Omega \cap \operatorname{Ker} L ; 0\right\} \\
= & \operatorname{deg}\left\{Q N\left(u_{1}, u_{2}, u_{3}, 1\right)^{T} ; \Omega \cap \operatorname{Ker} L ; 0\right\} \\
= & \operatorname{deg}\left\{Q N\left(u_{1}, u_{2}, u_{3}, 0\right)^{T} ; \Omega \cap \operatorname{Ker} L ; 0\right\} \\
= & \operatorname{sign}\left\{\operatorname{det}\left(\begin{array}{ccc}
\exp \left(u_{1}^{*}\right) & 0 & 0 \\
0 & \exp \left(u_{2}^{*}\right) & 0 \\
f_{1}\left(u^{*}\right) & 0 & f_{2}\left(u^{*}\right)
\end{array}\right)\right\},
\end{aligned}
$$

where

$$
\begin{aligned}
& f_{1}\left(u^{*}\right)=\frac{1}{\omega} \sum_{k=0}^{\omega-1}\left[\frac{d(k) \eta(k) \exp \left(u_{1}^{*}\right)\left(1+v_{1}(k) \exp \left(u_{3}^{*}\right)\right)}{\left(1+\omega_{1}(k) \exp \left(u_{1}^{*}\right)+v_{1}(k) \exp \left(u_{3}^{*}\right)\right)^{2}}\right]>0, \\
& f_{2}\left(u^{*}\right)=-\frac{1}{\omega} \sum_{k=0}^{\omega-1}\left[\frac{d(k) \eta(k) v_{1}(k) \exp \left(u_{1}^{*}+u_{3}^{*}\right)}{\left(1+\omega_{1}(k) \exp \left(u_{1}^{*}\right)+v_{1}(k) \exp \left(u_{3}^{*}\right)\right)^{2}}\right]<0 .
\end{aligned}
$$

Then, it follows from (3.62) that

$$
\operatorname{deg}\left\{J Q N\left(u_{1}, u_{2}, u_{3}\right)^{T} ; \Omega \cap \operatorname{ker} L ; 0\right\}=\operatorname{sign}\left[-\exp \left(u_{1}+u_{2}^{*}\right) f_{2}\left(u^{*}\right)\right]=-1<0 .
$$

By now, we have proved that $\Omega$ verifies all requirements of Lemma 3.1. Then it follows that $L u=N u$ has at least one solution in $\operatorname{Dom} L \cap \bar{\Omega}$, that is to say, (3.6) has at least one $\omega$-periodic solution in $\operatorname{Dom} L \cap \bar{\Omega}$, say $u^{*}=\left\{u^{*}(k)\right\}=\left\{\left(u_{1}^{*}(k), u_{2}^{*}(k), u_{3}^{*}(k)\right)^{T}\right\}$. Let $x_{1}^{*}(k)=\exp \left\{u_{1}^{*}(k)\right\}, x_{2}^{*}(k)=\exp \left\{u_{2}^{*}(k)\right\}$, and $x_{3}^{*}(k)=\exp \left\{u_{3}^{*}(k)\right\}$. Then we know that $\left(x_{1}^{*}(k), x_{2}^{*}(k), x_{3}^{*}(k)\right)^{T}$ is an $\omega$-periodic solution of system (2.3) with strictly positive components. We complete the proof.

\section{Acknowledgments}

This work is supported by National Natural Science Foundation of China (no. 10961008), the Scientific Research Fund of Hunan Provincial Education Department (no. 10C0560), and Doctoral Foundation of Guizhou College of Finance and Economics (2010).

\section{References}

[1] C. S. Holling, "The components of predation as revealed by a study of small mammal predation of the European pine sawfly," Canadian Entomologist, vol. 91, pp. 293-320, 1959. 
[2] W. Liu, D. Xiao, and Y. Yi, "Relaxation oscillations in a class of predator-prey systems," Journal of Differential Equations, vol. 188, no. 1, pp. 306-331, 2003.

[3] R. S. Cantrell, C. Cosner, and S. Ruan, "Intraspecific interference and consumer-resource dynamics," Discrete and Continuous Dynamical Systems. Series B, vol. 4, no. 3, pp. 527-546, 2004.

[4] W. Ko and K. Ryu, "Analysis of diffusive two-competing-prey and one-predator systems with Beddington-Deangelis functional response," Nonlinear Analysis. Theory, Methods \& Applications, vol. 71, no. 9, pp. 4185-4202, 2009.

[5] F. Chen, Y. Chen, and J. Shi, "Stability of the boundary solution of a nonautonomous predator-prey system with the Beddington-DeAngelis functional response," Journal of Mathematical Analysis and Applications, vol. 344, no. 2, pp. 1057-1067, 2008.

[6] H.-F. Huo, W.-T. Li, and J. J. Nieto, "Periodic solutions of delayed predator-prey model with the Beddington-DeAngelis functional response," Chaos, Solitons and Fractals, vol. 33, no. 2, pp. 505-512, 2007.

[7] H. K. Baek, "Qualitative analysis of Beddington-DeAngelis type impulsive predator-prey models," Nonlinear Analysis. Real World Applications, vol. 11, no. 3, pp. 1312-1322, 2010.

[8] R. S. Cantrell and C. Cosner, "On the dynamics of predator-prey models with the BeddingtonDeAngelis functional response," Journal of Mathematical Analysis and Applications, vol. 257, no. 1, pp. 206-222, 2001.

[9] X. Y. Song and Y. F. Li, “Dynamic complexities of a Holling II two-prey one-predator system with impulsive effect," Chaos, Solitons and Fractals, vol. 33, no. 2, pp. 463-478, 2007.

[10] J. R. Beddington, "Mutual interference between parasites or predators and its effect on searching efficiency," Journal of Animal Ecology, vol. 44, no. 1, pp. 331-340, 1975.

[11] D. L. DeAnglis, R. A. Goldstein, and R. V. O;Neil, "A model for trophic interaction," Ecology, vol. 56, no. 4, pp. 881-892, 1975.

[12] W. Ding and M. Han, "Dynamic of a non-autonomous predator-prey system with infinite delay and diffusion," Computers $\mathcal{E}$ Mathematics with Applications, vol. 56, no. 5, pp. 1335-1350, 2008.

[13] Y. Chen and Z. Zhou, "Stable periodic solution of a discrete periodic Lotka-Volterra competition system," Journal of Mathematical Analysis and Applications, vol. 277, no. 1, pp. 358-366, 2003.

[14] B. X. Dai and J. Z. Zou, "Periodic solutions of a discrete-time nonautonomous predator-prey system with the Beddington-DeAngelis functional response," Journal of Applied Mathematics \& Computing, vol. 24, no. 1-2, pp. 127-139, 2007.

[15] M. Fan and K. Wang, "Periodic solutions of a discrete time nonautonomous ratio-dependent predatorprey system," Mathematical and Computer Modelling, vol. 35, no. 9-10, pp. 951-961, 2002.

[16] M. Fazly and M. Hesaaraki, "Periodic solutions for a discrete time predator-prey system with monotone functional responses," Comptes Rendus Mathématique, vol. 345, no. 4, pp. 199-202, 2007.

[17] Y. K. Li, "Positive periodic solutions of a discrete mutualism model with time delays," International Journal of Mathematics and Mathematical Sciences, vol. 2005, no. 4, pp. 499-506, 2005.

[18] J. Wiener, "Differential equations with piecewise constant delays: Trends in theory and practice of nonlinear differential equations," in Lecture Notes in Pure and Applied Mathematics, vol. 90, Dekker, New York, NY, USA, 1984.

[19] R. Xu, L. Chen, and F. Hao, "Periodic solutions of a discrete time Lotka-Volterra type food-chain model with delays," Applied Mathematics and Computation, vol. 171, no. 1, pp. 91-103, 2005.

[20] H. Y. Zhang and Y. H. Xia, "Existence of positive periodic solution of a discrete time mutualism system with delays," Annals of Differential Equations, vol. 22, no. 2, pp. 225-233, 2006.

[21] J. M. Zhang and J. Wang, "Periodic solutions for discrete predator-prey systems with the BeddingtonDeAngelis functional response," Applied Mathematics Letters, vol. 19, no. 12, pp. 1361-1366, 2006.

[22] W. Zhang, D. Zhu, and P. Bi, "Multiple positive periodic solutions of a delayed discrete predator-prey system with type IV functional responses," Applied Mathematics Letters, vol. 20, no. 10, pp. 1031-1038, 2007.

[23] R. E. Gaines and J. L. Mawhin, Coincidence Degree and Nonlinear Differential Equations, Springer, Berlin, Germany, 1997. 


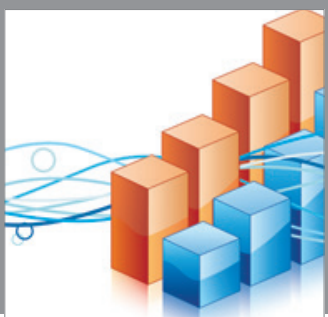

Advances in

Operations Research

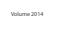

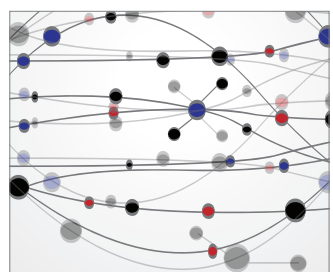

\section{The Scientific} World Journal
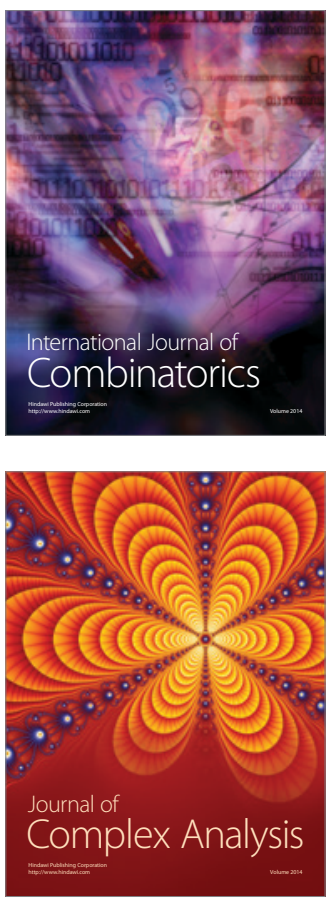

International Journal of

Mathematics and

Mathematical

Sciences
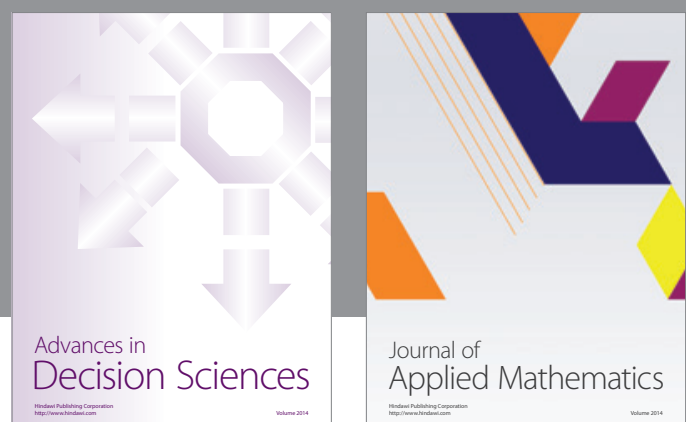

Journal of

Applied Mathematics
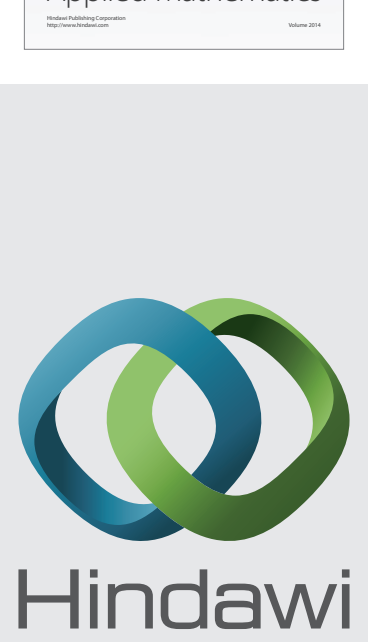

Submit your manuscripts at http://www.hindawi.com
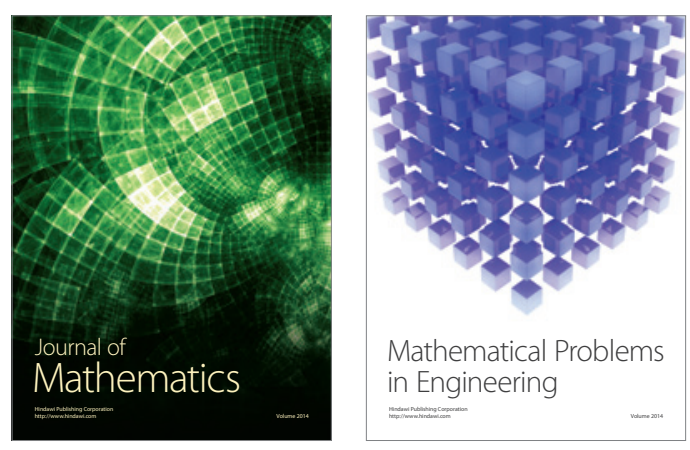

Mathematical Problems in Engineering
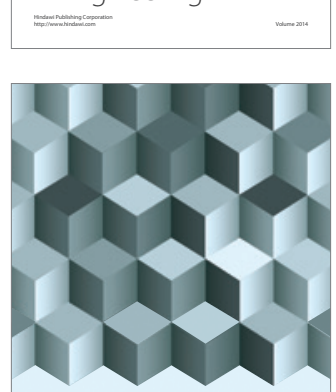

Journal of

Function Spaces
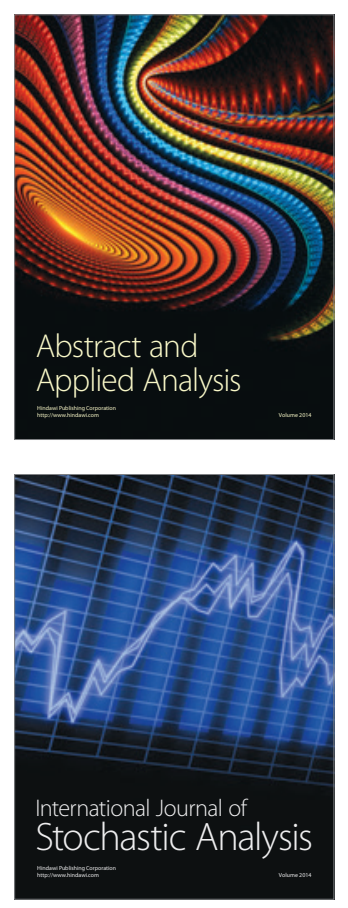

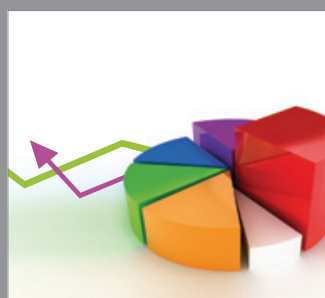

ournal of

Probability and Statistics

Promensencen
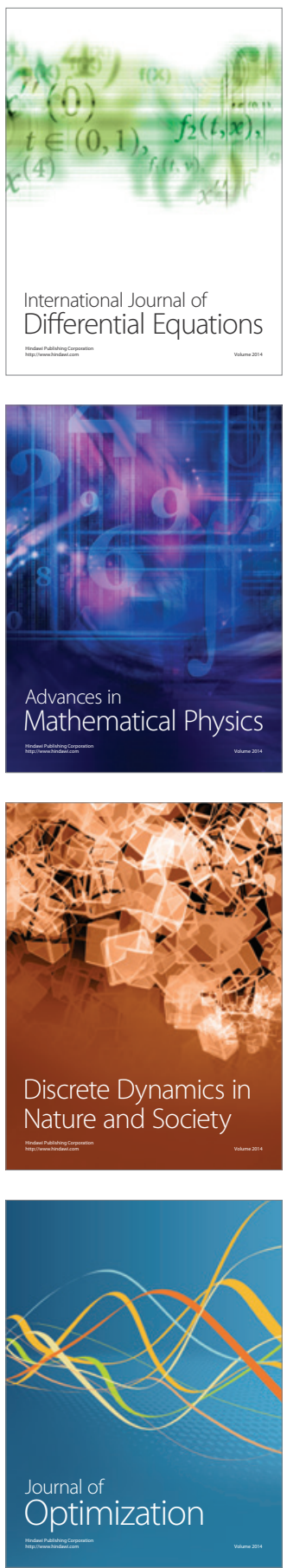